\title{
Stapler Closure versus Manual Closure in Total Laryngectomy for Laryngeal cancer: A Systematic Review and Meta-Analysis
}

\author{
Yi-Chan Lee ${ }^{1}$, Tuan-Jen Fang ${ }^{2}$, I-Chun Kuo ${ }^{3}$, Yao Te Tsai ${ }^{4}$, and Li-Jen Hsin ${ }^{2}$ \\ ${ }^{1}$ Chang Gung Memorial Hospital Keelung Branch \\ ${ }^{2}$ Chang Gung Memorial Hospital Linkou Main Branch \\ ${ }^{3}$ Chang Gung Memorial Hospital Linkou Branch \\ ${ }^{4}$ Chang Gung Memorial Hospital Chiayi Branch
}

May 14, 2020

\begin{abstract}
Abstract Objective: Total laryngectomy (TL) is a life-saving procedure for individuals with advanced laryngeal cancer or those suffering from recurrence after initial treatment. The present study aims to evaluate the differences between stapler closure (SC) and manual closure (MC) of the pharynx during TL for patients with laryngeal cancer. Design/Setting: A systematic literature search was performed using the PubMed, Embase and Cochrane Library. The data were analyzed using Comprehensive Meta-Analysis software (Version 3; Biostat, Englewood, NJ). Dichotomous data were calculated by odds ratio (OR) and continuous data were calculated by mean difference (MD) with 95\% confidence intervals (CI). Main outcome/Results: A total of 7 studies (535 patients) were included in this meta-analysis. Pooled analysis showed that the operative time of TL was significantly reduced in the SC group (MD, -63.2; 95\% CI, -106.0 to -20.4). Moreover, the SC group had a lower incidence of pharyngocutaneous fistula $(\mathrm{OR}=0.38 ; 95 \% \mathrm{CI}, 0.18-0.83 ; \mathrm{P}=0.016)$ and hospital stay $(\mathrm{MD},-2.9 ; 95 \% \mathrm{CI},-5.6$ to -0.1$)$. The incidence of postoperative surgical site infection $(\mathrm{OR}=0.41 ; 95 \% \mathrm{CI}, 0.02-8.73 ; \mathrm{P}=0.565)$ was comparable between the two groups. Conclusion: Based on these results, SC may be a useful option for patients who need TL.
\end{abstract}

\section{Introduction}

The role of radiation-based therapy in laryngeal cancer has been well established by several prospective randomized trials ${ }^{1-3}$. However, total laryngectomy (TL) is still one of the treatment options for advanced cases or when the disease fails to respond to initial organ preservation therapy. One of the critical steps in $\mathrm{TL}$ is the closure of the pharyngeal mucosa after the removal of the entire larynx. Adequate pharyngotomy closure should be tension-free and watertight to prevent leakage. In addition, the viability of the pharyngeal mucosa needs to be maintained as much as possible. Manual suturing by hand, though time-consuming, is the technique traditionally used for pharyngeal closure after TL. Since the development of surgical staplers in 1908, these mechanical suturing devices have been increasingly used to connect tissue in a sterile and efficient way ${ }^{4}$. Surgical stapler closure of the pharyngeal defect after laryngectomy was first reported in 1971 by a Russian study ${ }^{5}$. Several authors subsequently reported their experiences comparing the differences between manual closure (MC) and stapler closure (SC) of the pharyngeal defect after TL. The purpose of the present study was to compare intraoperative and postoperative parameters between $\mathrm{SC}$ and $\mathrm{MC}$ in total laryngectomy for laryngeal cancer in the existing English literature.

\section{Methods}

2.1 Literature search

This present analysis was conducted according to the Preferred Reporting Items for Systematic Reviews 
and Meta-Analyses statement ${ }^{6}$. Two of the authors (YCL and LJH) searched PubMed, EMBASE, and the Cochrane Library independently and extensively for articles of interest published before March 2020. The keywords used in the search process included "stapler" or "stapling" or "mechanical" and "laryngectomy". Moreover, reference lists of the included studies were also reviewed to identify additional studies.

\subsection{Study selection and data extraction}

The inclusion criteria were studies including only patients with laryngeal cancer who underwent TL, articles published in English, and studies comparing the outcomes of TL between the SC and MC techniques. The exclusion criteria were based primarily on the absence of one of the inclusion criteria. Studies without a control group, studies using the same database, articles not published in English, duplicate studies, case reports, abstracts, letters to the editor, and articles pending publication of the full text were excluded from the present analysis. Data were independently extracted by the 2 researchers (YCL and LJH). The bias in the studies was assessed independently by two researchers (YCL and LJH) using the Newcastle-Ottawa Scale and the Cochrane Collaboration's risk of bias tool (RoB 1.0) for nonrandomized and randomized studies, respectively 7,8 . Discrepancies in study bias classification were discussed between the two authors until consensus was achieved.

\subsection{Outcomes}

The main outcomes of this study included operative time, pharyngocutaneous fistula (PCF), length of hospitalization and postoperative surgical site infection.

\subsection{Data analysis}

The results were analyzed using Comprehensive Meta-Analysis software (Version 3; Biostat, Englewood, NJ). Mean differences (MDs) were calculated to compare the operative time and the length of hospitalization between the SC and MC groups. Odds ratios (ORs) were calculated to compare the PCF rate and postoperative surgical site infection rate between the SC and MC groups. The overall effect was pooled using a random-effects model. Statistical heterogeneity among studies was measured using the $I^{2}$ statistic, which calculated the proportion of overall variation attributable to between-study heterogeneity. An $I^{2}$ statistic exceeding $50 \%$ indicated moderate heterogeneity, and an $I^{2}$ statistic exceeding $75 \%$ indicated high heterogeneity ${ }^{9}$. Potential publication bias was assessed using a funnel plot and the Egger intercept test ${ }^{9}$. A 2 -sided $P$ value $<0.05$ was considered statistically significant.

\section{Results}

\subsection{Study selection}

The initial literature search yielded a total of 240 articles. Studies that were duplicated were excluded. Four articles were also excluded based on their titles and abstracts. The remaining 54 potentially eligible studies were retrieved for a careful review of the full texts. Among them, studies without a control group, review articles, studies with the same database, studies including non-laryngeal cancer patients, studies with unclear inclusion criteria and studies not written in English were excluded from the analysis. Finally, 7 articles were included in this review ${ }^{10-16}$. A flow diagram describing the process involved in study identification and inclusion/exclusion is shown in Figure 1. eTable 1 in the Supplement summarizes the literature search process and the keywords used.

\subsection{Demographics}

Table 1 lists the basic demographics of patients from the 7 articles, including two randomized studies and 5 nonrandomized studies. The pooled prevalences of preoperative tracheostomy and radiotherapy were both significantly higher in the $\mathrm{SC}$ group $(P=0.04$ and $P=0.00$, respectively). The bias assessment for each study is described in eTable 2 and eTable 3 in the Supplement.

\subsection{Outcomes}


3.3.1 Operative time Four of the included studies evaluated the operative time (total laryngectomy with/without neck dissection) of both procedures ${ }^{11,12,14,15}$. Two of these 4 studies included only cases of isolated total laryngectomy (without neck dissection) ${ }^{12,14}$. The pooled results of 4 studies showed that the operative time was lower in the SC group (MD, -63.2; 95\% confidence interval [CI], -106.0 to -20.4), and

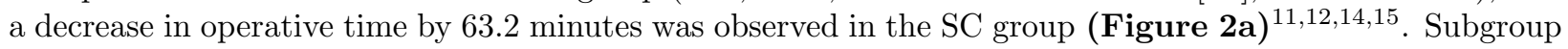
analysis including the 2 studies without neck dissection also showed that the operative time was lower in the SC group (MD, -45.9; 95\% CI, -64.2 to -27.6) with a decrease of 45.9 minutes $^{12,14}$. (Figure 2b). 3.3.2 PCF All of the included studies recorded the incidence of postoperative PCF in both groups. In the present study, the incidence rates of PCF were $13.7 \%$ (28/204) and 27.2\% (90/331) in the SC and MC groups, respectively. The pooled analysis showed that the PCF rate was lower in the SC group (OR $=0.38 ; 95 \%$ CI, $0.18-0.83 ; P$ $=0.016)$.(Figure 3). 3.3.3 Length of hospital stay Three of the included studies recorded the length of hospital stay in the two groups. The pooled analysis demonstrated that the length of hospital stay after TL was approximately 2.9 days shorter in the SC group than in the MC group (MD, -2.9; $95 \%$ CI, -5.6 to -0.1). (Figure 4). 3.3.4 Postoperative surgical site infection Two of the included studies recorded the incidence of postoperative surgical site infection in both groups. The pooled analysis showed no significant difference between the $\mathrm{SC}$ and $\mathrm{MC}$ groups regarding the rate of postoperative surgical site infection ( $\mathrm{OR}=0.41 ; 95 \%$ CI, 0.02-8.73; $P=0.565)$. (Figure 5).

\subsection{Publication Bias}

Funnel plots and the results of heterogeneity tests are presented ineFigure $\mathbf{1}$ in theSupplement . The plots of different parameters are generally symmetrical, suggesting no obvious publication bias. The results of the Egger intercept test also indicated no apparent publication bias.

\section{Discussion}

The present meta-analysis was conducted to evaluate the differences between the SC and MC techniques after TL for laryngeal cancer in the existing English literature. According to our meta-analysis, the SC group demonstrated a significantly shorter operative time than the $\mathrm{MC}$ group. In addition, we observed a lower incidence of PCF and hospital stay after SC. The differences in postoperative surgical site infection was not significant between groups with different closure techniques.

The pooled results from the analysis demonstrated that the operative time for TL is approximately 45.9 to 63.2 minutes shorter with the SC technique than with the MC technique. The result is intuitive because the surgical stapler device is capable of dividing and closing pharyngeal tissue simultaneously and instantly. In contrast, closure of the pharyngeal defect by suturing after removal of the larynx is a time-consuming step during TL. Ozturk et al reported that the closure time needed for the pharyngeal mucosa was only 3.0 minutes with a surgical stapler. However, it takes 37.5 minutes for surgeons to perform a watertight closure of the pharynx by hand ${ }^{16}$. Several studies also indicated that prolonged surgical time was significantly associated with a higher rate of postoperative complications in patients undergoing TL ${ }^{17-19}$. Pharyngeal closure with a surgical stapler can therefore be a time-saving technique for TL patients and potentially reduce the related complications. One important step in the SC technique is to ensure that the epiglottis is not trapped between the jaws of the surgical stapler. There are different strategies to solve this problem. Some authors retracted the epiglottis with an Allis clamp from the trachea lumen below with or without an endoscopic field of view ${ }^{10,11,13,15}$. Some suggested that a small midline pharyngotomy at the suprahyoid region enables the epiglottis to be pulled anteriorly under direct vision without tapping it in the pharyngeal mucosa ${ }^{14,20}$.

TL is a life-saving procedure for individuals diagnosed with advanced laryngeal cancer or those suffering from recurrence after organ preservation treatment ${ }^{21}$. However, permanent tracheostomy, speech difficulty and altered swallowing may adversely affect patients' physical and emotional health ${ }^{22}$. Among all the complications after TL, PCF is most commonly reported and is related to a prolonged hospital stay as well as delayed adjuvant therapy ${ }^{23}$. In the present meta-analysis, the incidence rates of PCF in the SC group and MC group were $13.7 \%$ (28/204) and 27.2\% (90/331), respectively. The incidence of PCF was significantly 
lower in the SC group. Two possible explanations exist for the reduced incidence of PCF in the SC group. First, six of the enrolled studies used the closed technique ${ }^{10-13,15,16}$, while the remaining study used the semiclosed technique ${ }^{14}$ when performing stapler closure of the pharynx. None of the SC groups in each study used the open technique, which involves the removal of the laryngeal specimen first and then the closure of the free edges of the pharyngotomy with a stapler. By avoiding the creation of a large pharyngeal defect before closure, the risk of surgical field contamination by pharyngeal secretions can be minimized with the use of a surgical stapler ${ }^{10,11,13}$. Second, blood supply to the surgical wound is believed to be fundamental to the healing process after TL. However, repeated trauma by forceps and focal ischemia from the tightening of sutures may both jeopardize the microcirculation of the pharyngeal mucosa during MC 10,11. On the other hand, the SC technique may be able to preserve more blood supply with simultaneous stapling as well as cutting of the pharynx, which may be beneficial to a better healing process. Radiotherapy ${ }^{24-27}$ and tracheostomy ${ }^{24,28}$ before TL have both been identified as independent factors that are associated with a higher rate of PCF. However, the pooled prevalence of patients receiving preoperative tracheostomy and radiotherapy was significantly higher in the $\mathrm{SC}$ group. The pooled results implied that the $\mathrm{SC}$ technique has the potential to decrease the rate of PCF formation after TL even in irradiated or tracheostomized patients.

The pooled results demonstrated that the length of hospital stay after TL was approximately 2.9 days shorter in the SC group than in the MC group and this probably reflected the different rate of postoperative PCF between the two groups. Because of the lower PCF incidence with the SC technique, more patients may be able to try oral feeding earlier and reduce their length of hospital stay. Using a surgical stapler to close pharyngeal defects during TL can prevent pharyngeal content from contaminating the operative field. Theoretically, a reduced surgical site infection rate is expected in the SC group. However, the results of the present meta-analysis did not support this idea, and the rate of surgical site infection was comparable between the SC and MC groups. One possible reason may be that surgical site infection is a multifactorial complication. Factors such as the general health status of the patient, a lower serum albumin level, perioperative blood transfusion and operation time can all be associated with the rate of infection ${ }^{29,30}$.

This study had several limitations. First, only seven studies were included in this meta-analysis. More studies are needed to confirm the pooled results. Second, although some of the included articles were randomized studies, retrospective studies were also included because of the lack of available data in the current literature. Third, the analysis of funnel plots and Egger's tests demonstrated no obvious evidence of publication bias. However, the results of this meta-analysis should be interpreted with caution because of the heterogeneity between the included studies. Despite these limitations, our meta-analysis still provides evidence for the use of different closure techniques in TL.

\section{Conclusion}

In conclusion, the SC technique significantly reduced the operative time needed for TL. In addition, we also observed a lower incidence of PCF and hospital stay after SC in all patients even though there were more irradiated and tracheostomized patients in the SC group. We found similar surgical site infection rates between the SC and MC groups. The presented meta-analysis provides current evidence for the use of SC in laryngeal cancer patients undergoing TL. The surgical stapler has the potential to be a useful tool in the pharyngeal closure step during TL.

\section{Key points}

* Total laryngectomy is a life-saving procedure for individuals with advanced laryngeal cancer or those suffering from recurrence after initial treatment.

* Manual suturing of the pharyngeal mucosa after the removal of the entire larynx, though time-consuming, is the technique traditionally used for pharyngeal closure after total laryngectomy.

* The stapler closure technique significantly reduced the operative time needed for total laryngectomy. In addition, we observed a lower incidence of pharyngocutaneous fistula and hospital stay after stapler closure in all patients. The rate of surgical site infection was comparable between the stapler closure and manual 
closure groups.

* The presented meta-analysis provides current evidence for the use of stapler closure in laryngeal cancer patients undergoing total laryngectomy. The surgical stapler has the potential to be a useful tool in the pharyngeal closure step during total laryngectomy.

\section{References}

1. Lefebvre JL, Andry G, Chevalier D, et al. Laryngeal preservation with induction chemotherapy for hypopharyngeal squamous cell carcinoma: 10-year results of EORTC trial 24891. Ann Oncol.2012;23(10):27082714.

2. Forastiere AA, Zhang Q, Weber RS, et al. Long-term results of RTOG 91-11: a comparison of three nonsurgical treatment strategies to preserve the larynx in patients with locally advanced larynx cancer. $J$ Clin Oncol. 2013;31(7):845-852.

3. Department of Veterans Affairs Laryngeal Cancer Study G, Wolf GT, Fisher SG, et al. Induction chemotherapy plus radiation compared with surgery plus radiation in patients with advanced laryngeal cancer. N Engl J Med. 1991;324(24):1685-1690.

4. Gaidry AD, Tremblay L, Nakayama D, Ignacio RC, Jr. The History of Surgical Staplers: A Combination of Hungarian, Russian, and American Innovation. Am Surg. 2019;85(6):563-566.

5. Luk'ianchenko AG. [Suturing of a laryngeal defect in laryngectomy]. Vestn Otorinolaringol. 1971;33(6):2931.

6. Moher D, Liberati A, Tetzlaff J, Altman DG, Group P. Preferred reporting items for systematic reviews and meta-analyses: the PRISMA statement. PLoS Med. 2009;6(7):e1000097.

7. Higgins JP, Altman DG, Gotzsche PC, et al. The Cochrane Collaboration's tool for assessing risk of bias in randomised trials. BMJ. 2011;343:d5928.

8. Peterson J, Welch V, Losos M, Tugwell PJOOHRI. The Newcastle-Ottawa scale (NOS) for assessing the quality of nonrandomised studies in meta-analyses. 2011.

9. Higgins JP, Thompson SG, Deeks JJ, Altman DG. Measuring inconsistency in meta-analyses. BMJ. 2003;327(7414):557-560.

10. Goncalves AJ, de Souza JA, Jr., Menezes MB, Kavabata NK, Suehara AB, Lehn CN. Pharyngocutaneous fistulae following total laryngectomy comparison between manual and mechanical sutures. Eur Arch Otorhinolaryngol. 2009;266(11):1793-1798.

11. Calli C, Pinar E, Oncel S. Pharyngocutaneous fistula after total laryngectomy: Less common with mechanical stapler closure. Ann Otol Rhinol Laryngol. 2011;120(5):339-344.

12. Sannikorn P, Pornniwes N. Comparison of outcomes for staple and conventional closure of the pharynx following total laryngectomy.J Med Assoc Thai. 2013;96 Suppl 3:S89-93.

13. Dedivitis RA, Aires FT, Pfuetzenreiter EG, Jr., Castro MA, Guimaraes AV. Stapler suture of the pharynx after total laryngectomy. Acta Otorhinolaryngol Ital. 2014;34(2):94-98.

14. Ismi O, Unal M, Vayisoglu Y, et al. Stapler Esophageal Closure During Total Laryngectomy. J Craniofac Surg. 2017;28(1):e35-e40.

15. Galletti B, Freni F, Catalano N, et al. Linear stapler closure of the pharynx in laryngectomy: our experience (endoscopic closed technique). 2018.

16. Ozturk K, Turhal G, Ozturk A, Kaya I, Akyildiz S, Uluoz U. The Comparative Analysis of Suture versus Linear Stapler Pharyngeal Closure in Total Laryngectomy: A Prospective Randomized Study. Turk Arch Otorhinolaryngol. 2019;57(4):166-170. 
17. Helman SN, Brant JA, Kadakia SK, Newman JG, Cannady SB, Chai RL. Factors associated with complications in total laryngectomy without microvascular reconstruction. Head Neck. 2018;40(11):24092415 .

18. Schwartz SR, Yueh B, Maynard C, Daley J, Henderson W, Khuri SF. Predictors of wound complications after laryngectomy: A study of over 2000 patients. Otolaryngol Head Neck Surg. 2004;131(1):61-68.

19. Helman SN, Brant JA, Moubayed SP, Newman JG, Cannady SB, Chai RL. Predictors of length of stay, reoperation, and readmission following total laryngectomy. Laryngoscope. 2017;127(6):1339-1344.

20. Altissimi G, Frenguelli A. Linear stapler closure of the pharynx during total laryngectomy: a 15-year experience (from closed technique to semi-closed technique). Acta Otorhinolaryngol Ital.2007;27(3):118-122.

21. Goepfert RP, Hutcheson KA, Lewin JS, et al. Complications, hospital length of stay, and readmission after total laryngectomy. Cancer.2017;123(10):1760-1767.

22. Lefebvre JL. Laryngeal preservation in head and neck cancer: multidisciplinary approach. Lancet Oncol. $2006 ; 7(9): 747-755$.

23. Cavalot AL, Gervasio CF, Nazionale G, et al. Pharyngocutaneous fistula as a complication of total laryngectomy: review of the literature and analysis of case records. Otolaryngol Head Neck Surg. 2000;123(5):587592 .

24. Kim YH, Roh JL, Choi SH, Nam SY, Kim SY. Prediction of pharyngocutaneous fistula and survival after salvage laryngectomy for laryngohypopharyngeal carcinoma. Head Neck. 2019;41(9):3002-3008.

25. Busoni M, Deganello A, Gallo O. Pharyngocutaneous fistula following total laryngectomy: analysis of risk factors, prognosis and treatment modalities. Acta Otorhinolaryngol Ital. 2015;35(6):400-405.

26. Boscolo-Rizzo P, De Cillis G, Marchiori C, Carpene S, Da Mosto MC. Multivariate analysis of risk factors for pharyngocutaneous fistula after total laryngectomy. Eur Arch Otorhinolaryngol.2008;265(8):929-936.

27. Klozar J, Cada Z, Koslabova E. Complications of total laryngectomy in the era of chemoradiation. Eur Arch Otorhinolaryngol.2012;269(1):289-293.

28. Benson EM, Hirata RM, Thompson CB, et al. Pharyngocutaneous fistula after total laryngectomy: a single-institution experience, 2001-2012.Am J Otolaryngol. 2015;36(1):24-31.

29. Lee Y-J, Wang C-C, Wang C-P, Jiang R-S, Liu S-AJ. Risk Factors of Surgical Site Infection After Total Laryngectomy. 2013;48(1):45-54.

30. Gonzalez Aguilar O, Pardo HA, Vannelli A, et al. Total laryngectomy: pre- and intrasurgical variables of infection risk. Int Surg.2001;86(1):42-48.

\section{Acknowledgments}

We thank Alfred Hsing-Fen Lin, MS, Raising Statistics Consultant Inc, for his statistical assistance. He received compensation and declared no competing interests between this study and his company.

\section{Hosted file}

Table 1.docx available at https://authorea.com/users/321855/articles/451042-stapler-closureversus-manual-closure-in-total-laryngectomy-for-laryngeal-cancer-a-systematic-reviewand-meta-analysis 


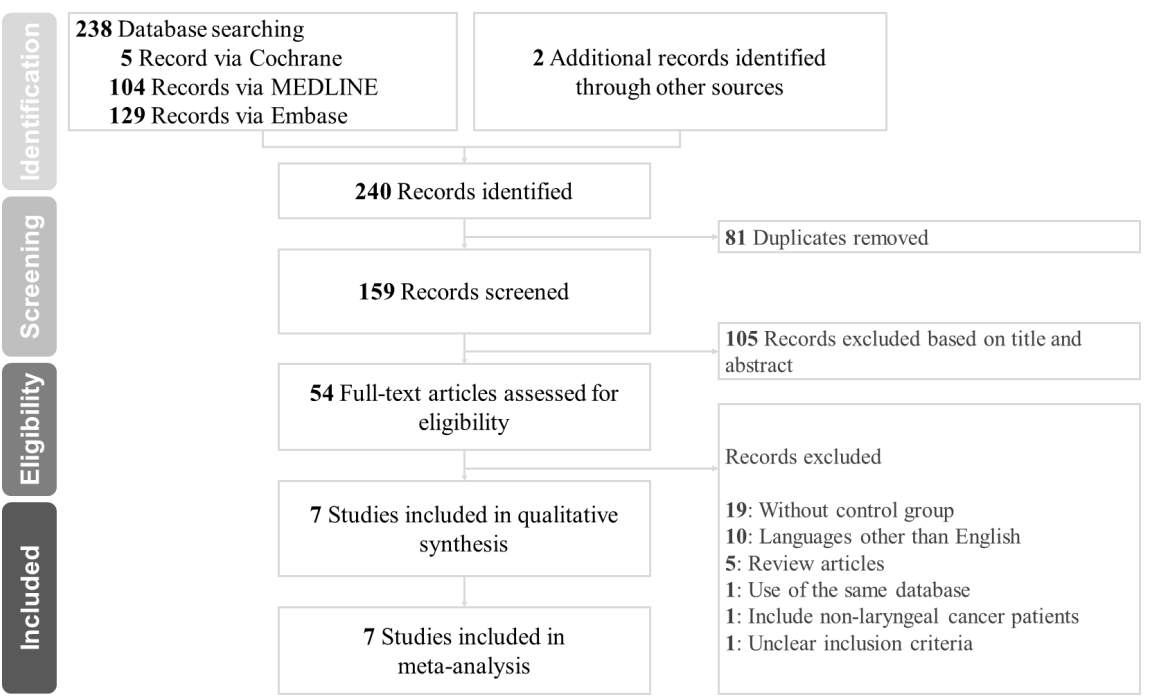

a

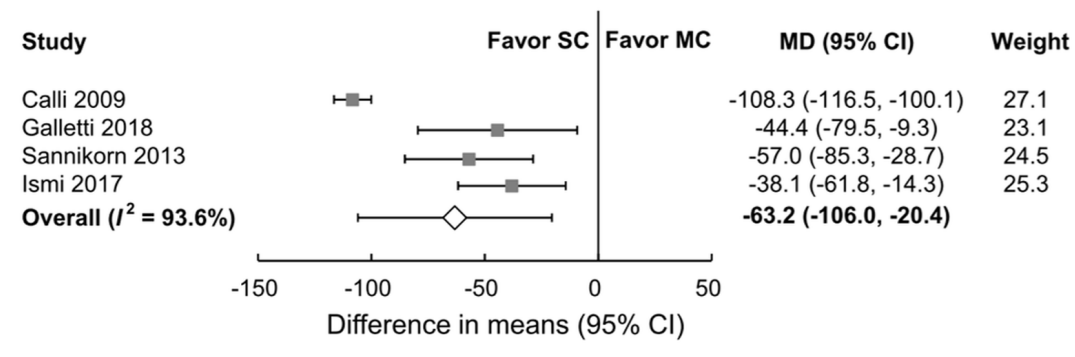

b

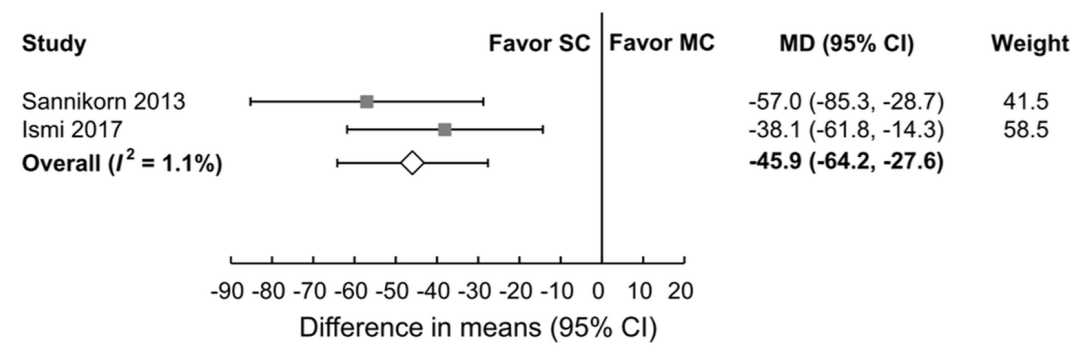



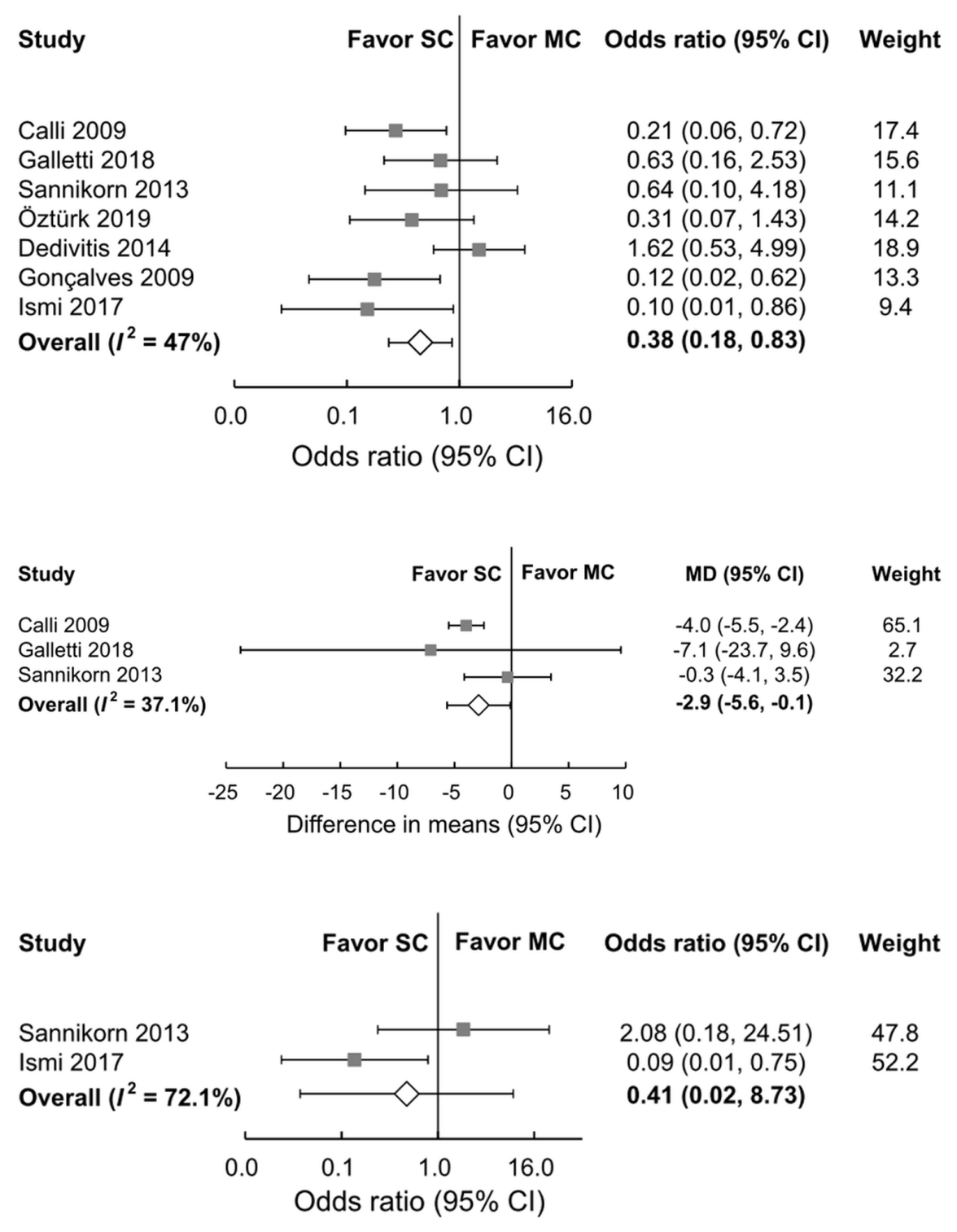